\title{
Genetic Analysis and Gene Mapping of Two Whitebacked Planthopper Resistance Genes From Rice Varieties
}

\section{Ling Cheng}

Yangtze University

\section{Baoxuan Nong}

Guangxi Academy of Agricultural Science

\section{Anlong Xu}

Guangxi University

Wenai He

Guangxi University

\section{Biqiu Wu}

Guangxi Academy of Agricultural Science

\section{Yongfu QIU ( $\square$ yfqiu@126.com )}

Guangxi University

\section{Research}

Keywords: whitebacked planthopper (WBPH), both brown planthopper (BPH)

Posted Date: April 23rd, 2021

DOl: https://doi.org/10.21203/rs.3.rs-448706/v1

License: (9) (i) This work is licensed under a Creative Commons Attribution 4.0 International License. Read Full License 
1 Genetic analysis and gene mapping of two whitebacked planthopper resistance

2 genes from rice varieties

3

4 Ling Cheng ${ }^{1,+}$, Baoxuan Nong ${ }^{3,+}$, Anlong $\mathrm{Xu}^{3}$, Wenai $\mathrm{He}^{3}$, Biqiu $\mathrm{Wu}^{2 *}$, Yongfu $\mathrm{Qiu}^{4 *}$ 5

6 Author affiliation:

71 College of Agriculture, Yangtze University, Jingzhou 434025, China

82 Plant Protection Institute, Guangxi Key Laboratory of Biology for Crop Diseases

9 and Insect Pests, Guangxi Academy of Agricultural Science, Nanning 530007, China

103 Rice Research Institute, Guangxi Key Laboratory of Rice Genetics and Breeding,

11 Guangxi Academy of Agricultural Science, Nanning 530007, China

124 Agricultural College, Guangxi University, Nanning 530004, China

13

$14+$ These authors contributed equally.

15

16 Corresponding author: wubiq@126.com, yfqiu@126.com 


\section{Abstract \\ Background}

The whitebacked planthopper $(\mathrm{WBPH})$ has become a significant threat to rice production. Identification of WBPH-resistant germplasm and genes can drive efforts to develop resistance varieties and effectively limit pest damage.

\section{Methods}

Fourteen varieties of rice were surveyed for insect resistance using tests that assessed bulk seedling growth rates, insect feeding activity (via measurements of honeydew excretion weight), and insect development (by counting the number of hatched nymphs). Two resistance varieties N22 and OB677 were crossed with susceptible line 9311 to develop mapping populations, which were applied to map the resistance genes/QTLs.

\section{Results}

The rice variety PTB33 showed high resistance to both brown planthopper (BPH) and WBPH, and varieties N22, RBPH327, and OB677 showed moderate resistance to WBPH. Host choice test and seedling survival rates further verified the WBPH resistance of PTB33, N22, and OB677. By using two $\mathrm{F}_{2}$ mapping populations, two major resistance genes were detected in N22 and OB677. Wbph1 was mapped on chromosome 2 of N22 in a region that harbored the markers RM13650 and RM13478. Its largest logarithm of the odds (LOD) score was 3.94, which explained a 16.6\% phenotypic variation. Wbph9 was mapped on chromosome 3 of OB677, where it was flanked by markers RM3513 and RM3525. It had a LOD score of 3.4, explaining a $17.2 \%$ phenotypic variation.

\section{Conclusions}

Four varieties PTB33, N22, RBPH327, and OB677 showed resistance to WBPH, of which OB677 was a novel resistance germplasm; and a novel resistance gene Wbph9 was mapped on chromosome 3. 


\section{Background}

The white-backed planthopper (WBPH), Sogatella furcifera (Horváth), is a major pest that affects rice crops throughout rice-growing regions worldwide, especially in Asia-Pacific countries. The frequency of WBPH outbreaks has increased markedly in recent years, causing severe drops in rice production (Hu et al, 2015). The WBPH causes damage to rice crops by feeding, ovipositing, and transmitting viruses. Specifically, it feeds by directly sucking on the phloem sap, reducing crop yields and even killing the plants. In addition, the WBPH can cause indirect damage by acting as vectors for transmitting viral diseases. These viruses include the rice grassy stunt viruses (GSV) and ragged stunt viruses (RSV) (Anjaneyulu, 1986) that cause plant dwarfism and reduce tillers. This indirect viral-mediated damage has caused huge losses in rice production in Asia countries. For instance, more than 700,000 ha in 2011 and 500,000 ha in 2012 were seriously suffered Southern rice black-streaked dwarf virus (SRBSDV) transmitted by WBPH in Vietnam and China, respectively (Zhou et al, 2013). It has been difficult to manage WBPHs using traditional chemical applications due to its long-distance migration and quick development of resistance against pesticides. Instead, host plant resistance is considered an efficient and environmentally friendly way to control WBPH infestation, and natural planthopper resistance genes could be exploited for developing novel resistant varieties. Therefore, the identification of WBPH-resistant rice germplasm and the corresponding resistance genes is an important and necessary task that can contribute to rice breeding programs.

To date, 12 major WBPH resistance genes have been identified in various cultivars or wild rice species (Du et al. 2020). Specifically, Wbphl was first detected in the rice variety N22 (Sidhu and Khush, 1979); and further studies indicated that Wbph1 co-segregated with the restriction fragment length polymorphism (RFLP) markers RG146 and RG445 in variety IR36 (McCouch et al, 1991). Wbph2 was identified in variety ARC10239 and mapped to chromosome 6 where it was associated with RFLP markers RZ667, RG264, and RG64 (Angeles et al, 1981; Liu et al, 2002). Wbph3 and wbph4 were detected in varieties ADR52 and Podiwi A8, respectively 
(Hernandez and Khush, 1981). Wbph5 was detected in variety N'Diang Marie (Wu and Khush, 1985), and Wbph6(t) was associated with RM167 on chromosome 11 (Li et al, 1990; Li et al, 2004). Wbph7 and Wbph8 were derived from introgressed lines of O. officinalis and were mapped to the same chromosomal regions as the $\mathrm{BPH}$ resistance genes Bph14 and Bph15, respectively (Tan et al, 2004). Further study indicated that Bph14 confers resistance to both BPH and WBPH (Tan et al, 2004). In summary, Wbph1, Wbph3, wbph4, and Wbph5 were not assigned to any chromosomes, and Wbph2 and Wbph6 were only roughly mapped onto the chromosomes and no tightly linked markers or convenient markers were available for marker-assisted selection (MAS). Several quantitative trait loci (QTL) associated with WBPH resistance were also detected and mapped from variety Chunjiang 06 and Dongxiang wild species (Sogawa et al, 2005; Chen et al, 2010). Currently, only very few WBPH resistance genes have been detected and finely mapped. There are several cloned BPH resistance genes such as $B p h 6, B p h 14$, and $B p h 3$ that simultaneously confer resistance to BPH and WBPH (Tan et al. 2004; Liu et al. 2016; Guo et al. 2018). It is therefore also important to evaluate the WBPH resistance of varieties that already carry $\mathrm{BPH}$ resistance genes, and through which we can detect more double resistance rice germplasm.

In this study, the purpose was to evaluate the planthoppers resistance level of collected rice varieties which carry BPH or WBPH resistance genes using the insect populations collected from rice paddy in Nanning, Guangxi; and to map the WBPH resistance genes from the detected resistance rice varieties. In detail, six rice varieties that carry BPH resistance genes and four varieties that carry WBPH resistance genes were surveyed for WBPH resistance using the bulk seedling test and by assessing WBPH feeding and development. Two resistant varieties, N22 and OB677, were subject to further resistance gene mapping and genetic analyses as $F_{2}$ mapping populations. As a result, two WBPH resistance genes, Wbph1 and Wbph9, were discovered, and assigned to chromosomes 2 and 3 respectively, with corresponding molecular markers.

\section{Materials and methods}


106 Fourteen rice varieties were used in the study (Table 1). Varieties N22, Baiganruo,

107 Nebeshi, and Cdombo were reported to be resistant to WBPH and carried the resistance genes Wbph1, Wbph2, Wbph3, and wbph4, respectively (Sidhu et al, 1979; Angeles et al, 1981; Hernandez and Khush, 1981). Varieties Mudgo, ASD7, RathuHeenati, and ARC 10550 were reported to carry the resistance genes Bph1, bph2, $B p h 3$, and bph5, respectively (Athwal et al, 1971; Lakshminarayana \& Khush, 1977; Khush et al, 1985; Nemoto et al, 1989). Despite carrying these genes, in 2006, Mudgo, ASD7, and ARC 10550 were found to be susceptible to BPH insect populations collected from rice fields in Wuhan, China (Qiu et al, 2011). It remains unclear whether the rice variety OB677 carries any planthopper resistance genes. The ten varieties N22, Baiganruo, Nebeshi, Cdombo, Mudgo, ASD7, RathuHeenati, ARC 10550, OB677, and PTB33 were collected from the China National Rice Research Institute or International Rice Research Institute (IRRI). Rice lines RBPH16 and RBPH327 were derived from the common wild rice species $O$. rufipogon Griff, and identified to be resistant to BPH and WBPH (Qiu et al, 2012; Yang et al, 2020). Varieties 9311 and TN1 were highly susceptible to BPH and WBPH.

WBPH and BPH insects were collected from rice fields in Nanning of Guangxi province, China $\left(22^{\circ} 49^{\prime} \mathrm{N}, 108^{\circ} 19^{\prime} \mathrm{E}\right)$, and reared on $\mathrm{TN} 1$ seedlings in a greenhouse under natural light at $26-30^{\circ} \mathrm{C}$ in Guangxi University. Second to third instar nymphs or adults were used in experiments. All tests were conducted in a greenhouse under natural light at $26-30^{\circ} \mathrm{C}$ from 2014 to 2017.

\section{Evaluation of insect resistance}

The bulk seedling growth test was performed to evaluate WBPH and BPH resistance. Germinated seeds were sown in a plastic tray $(48 \times 39 \times 7 \mathrm{~cm})$ to obtain 16 seedlings per row. A total of 10 tested rows were sown in one tray and one row of TN1 (susceptible control) was randomly sown among the tested varieties. At the second-leaf stage (approximately 7 days old), the seedlings were infested with $2^{\text {nd }}-3^{\text {rd }}$ WBPH instars at 10 insects per seedling and covered with a fine, light-transmitting mesh $(44 \times 34 \times 44 \mathrm{~cm})$. To evaluate $\mathrm{BPH}$ resistance, the same treatment was designed 
except that third-leaf seedlings were infested with eight nymphs per plant. When all of the control TN1 plants had died (approximately 15 days for WBPH and 9 days for BPH after infestation), each seedling was assigned a score of $0,1,3,5,7$, or 9 according to the criteria for the Standard Evaluation System for Rice (IRRI, 1988; Qiu et al, 2010). The average resistance score of each variety was calculated from the scores of all seedlings. Both tests were performed three independent times.

To survey WBPH host choice and seedling survival rates, each plastic tray $(32 \times 26 \times 12 \mathrm{~cm})$ was divided into four approximately equal portions and filled with soil to a height of $8 \mathrm{~cm}$. There were approximately 300 total numbers of seedlings per tray, with 70 to 90 of each variety. The two-leaf stage seedlings (approximately 7 days old) were infested with 100 female adults, and covered with a fine, light-transmitting mesh $(44 \times 34 \times 44 \mathrm{~cm})$. The numbers of nymphs on the seedlings were recorded 10 days later, and the numbers of surviving seedlings were recorded 30 days after infestation. The test was repeated twice and two replicates were designed for each. As there had too much individuals and the nymphs was relatively small, it was hard to exactly count the number. Therefore, more than five seedlings from four locations of each variety were selected and recorded.

\section{Honeydew excretion quantity}

To assess the feeding activity of WBPH, we quantified honeydew excreted by each insect. An average of four 7 day-old seedlings was planted uniformly in each bucket ( $25 \mathrm{~cm}$ in diameter, $15 \mathrm{~cm}$ in height) that was filled with soil to a height of $12 \mathrm{~cm}$. Each WBPH insect with a short wing was released into a rectangular pre-weighed parafilm sachet $(3.5 \mathrm{~cm}$ length, $3 \mathrm{~cm}$ width) that had been fastened onto a 35 day-old rice shoot (Pathak et al, 1982; Smith, 1994). Two days after infestation, each sachet containing excreted honeydew was re-weighed and the weight of the sachet alone subtracted to give the net weight of honeydew excreted. For the 14 varieties studied, eight plants of each variety were surveyed for honeydew excretion using two bags per plant and the test was repeated twice. For plants from the $F_{2}$ population, four bags per plant were used and the same honeydew excretion test was applied. Each test was repeated once on the same plant after one week. 


\section{Development of mapping population and gene mapping}

166

167

168

The varieties N22 and OB677 were crossed with 9311 to obtain $F_{1}$ individuals which were self-pollinated to develop two $\mathrm{F}_{2}$ mapping populations, 9311/N22 and 9311/OB677 in 2013; and then they were used for genetic analysis and gene mapping. As the bulk seedling test is approximate and subjective, it can be difficult to determine the precise resistance scores for each seedling. Furthermore, it was difficult to obtain fresh leaf samples for genotyping after infestation, especially when two-leaf seedlings were used and many had started to die. Therefore, the quantity of WBPH honeydew excreted after feeding on $\mathrm{F}_{2}$ individuals was taken as the phenotypic index for resistance gene mapping. To perform gene mapping, bulked segregant analysis was applied to detect molecular markers that were associated with WBPH resistance (Michelmore et al, 1991). Specifically, two contrasting bulk DNA samples, collected from plants that produced the ten largest or least WBPH honeydew excretion weights, were used to screen the SSR or InDel marker from 12 rice chromosomes. The polymorphic markers detected between two bulk DNA samples were most possibly linked with WBPH resistance gene. Then, more polymorphic markers between parents around the linked markers were obtained and applied to analyze the genotypes of the $\mathrm{F}_{2}$ population. After this was performed, one local genetic linkage map of the resistance gene containing region was generated by performing JoinMap 3.0 with detected genotypes (Van Ooijen, 2001). Finally, a permutation test (1000 times) was applied to calculate the logarithm of odds (LOD) threshold, and the resistance effect of the interested region was analyzed by MapQTL 5 (Van Ooijen and Voorrips, 2004). The locus with a LOD score > 3.0 was reported as one QTL and the position with the largest LOD score was taken as the target gene.

\section{Statistical analysis}

Data were analyzed using one-way ANOVA and means were compared using a least significant difference test with SPSS 13.0 (SPSS Institute Inc, Chicago, IL, USA). The seedling survival rates (\%) were arcsine transformed prior to analysis.

\section{Results}

\section{Evaluation of WBPH resistance}


The average WBPH resistance scores of the 14 rice varieties detected in the bulk seedling test ranged from 3.2 to 9.0 (Table 1). According to the resistance evaluation criteria, varieties N22, PTB33, RBPH327, and OB677 showed high resistance to WBPH (<4.2); whereas varieties Baiganruo, Mudgo, ASD7, ARC10550, 9311, and TN1 were highly susceptible (>7.0). The other six tested varieties showed moderate resistance to WBPH. The BPH resistance scores of PTB33, Babawee, RBPH16, and RBPH327 were less than 4.0, indicating strong resistance to BPH. Variety OB677 had moderate resistance to $\mathrm{BPH}$ (5.2). In contrast, the resistance scores of all other nine varieties tested were $>8.0$ and exhibited high susceptibility to BPH (Table 1).

The quantity of honeydew excreted by each WBPH was measured after two days of infestation to assess its feeding activity on the different rice varieties. There was a large variation in honeydew excretion (Table 1). The amount of honeydew excretion was the lowest on variety PTB33 by WBPH feeding $(0.2 \mathrm{mg})$. Higher amounts $(>8.0$ mg) of honeydew were excreted by WBPH feeding on varieties ASD7 (8.4 mg), 9311 (12.6 mg), and TN1 (9.2 mg). Less than $3.0 \mathrm{mg}$ of honeydew was excreted by WBPH feeding on N22 (1.4 mg), Babawee (1.2 mg), OB677 (1.2 mg), RBPH16 (2.2 mg), and RBPH327 (2.1 mg) varieties (Table 1).

To observe WBPH growth and development on the different rice varieties, we counted the numbers of hatched nymphs. The number of hatched nymphs varied greatly across the plant varieties. Less than 10 nymphs were observed on N22 (8), PTB33 (5), Babawee (6), and RBPH16 (9) varieties. In contrast, more than 30 nymphs were detected on Nebeshi (32), ASD7 (36), ARC10550 (32), 9311 (52), and TN1 (50) varieties (Table 1).

\section{Verifying the resistance of varieties $\mathrm{N} 22$ and $0 \mathrm{OB677}$}

Insect host choice and seedling survival rates were surveyed to verify the resistance of N22 and OB677 to WBPH. Variety PTB33 was taken as a resistance control (Fig. 1a, b). Significantly more WBPH nymphs were observed on the susceptible plants at 10 days after infestation (Fig. 1c). Specifically, there were 16, 4, 5, and 1 nymph(s) per plant on 9311, N22, OB677, and PTB33, respectively (Fig. 1e). There were 9311 plants that were almost dead at 30 days after infestation and its average survival rate 
was 2.3\%; while the survival rates of N22, OB677, and PTB33 were 83.6\%, 74.7\%, and 98.2\%, respectively (Fig. 1d, f). These results indicated that PTB33 showed high resistance to $\mathrm{WBPH}$; whereas $\mathrm{N} 22$ and $\mathrm{OB} 677$ were moderately resistance to these insects.

\section{Distribution of honeydew excretion by WBPH feeding on the $\mathrm{F}_{2}$ plants}

The weight of honeydew excreted varied from $0.3 \mathrm{mg}$ to $13.1 \mathrm{mg}$ in WBPH feeding on the $F_{2}$ rice populations of $9311 / \mathrm{N} 22$, and most weights were clustered in the region of 3.1-5.0 mg (Fig. 2a). The weight of honeydew excreted also varied in the $F_{2}$ population of 9311/OB677 and ranged from $0.4 \mathrm{mg}$ to $11.3 \mathrm{mg}$. No significant peaks of specific regions were observed in the mapping population (Fig. 2b). The phenotypic distributions suggested that the WBPH resistance was possibly controlled by one or more QTLs in the resistant varieties.

\section{Identification of the WBPH resistance gene in rice varieties $\mathbf{N 2 2}$ and $0 B 677$}

Two chromosomal regions were detected to be polymorphic in the two bulk DNA samples that were checked by a total of 1120 makers. One region was located on the long arm of chromosome 2 of N22, and the other was on chromosome 3 of OB677 (Table 2). The two local genetic linkage groups identified were $21.1 \mathrm{cM}$ and $11.6 \mathrm{cM}$ for N22 and OB677, respectively. The marker orders were in accordance with the genome of variety Nipponbare

(http://rice.plantbiology.msu.edu/cgi-bin/gbrowse/rice/). Interval mapping with MapQTL 5 was then used to analyze the genotype and phenotype of the $\mathrm{F}_{2}$ individuals. Subsequently, one major QTL with the largest LOD score of 3.94 was detected between RM13650 and RM13478, which explained the 16.6\% phenotypic variation in the 9311/N22 $\mathrm{F}_{2}$ mapping population. This was designated Wbph1 as the gene had been previously identified in N22 (Sidhu and Khush, 1979). Another QTL was harbored with markers RM3513 and RM3525, which had a LOD score of 3.4, and explained the $17.2 \%$ phenotypic variation in the $9311 / \mathrm{OB} 677 \mathrm{~F}_{2}$ mapping population. The detected QTL was designated Wbph9 according to the rules for nomenclature (Yamazaki et al. 2010:http://www.shigen.nig.ac.jp/rice/oryzabase/), as no WBPH resistance gene in this region had been previously reported. 


\section{Discussions}

WBPH has become a menace to rice production, especially due to its transmission of viruses such as GSV and RSV that cause stunting, lower yields and plant death. A non-chemical approach to manage WBPH infestation is to identify WBPH resistant germplasm and resistance genes to breed resistant varieties. Fortunately, over the years, researchers have been prioritizing detecting resistance varieties and lines, contributing to this research effort. For instance, Cao et al. (1993) screened 25, 545 local varieties for WBPH resistance and obtained 437 varieties with resistance levels of less than 1.0. Huang et al. (2012) surveyed 383 rice lines from the IRRI and found 107 lines that were resistant to both BPH and WBPH. However, while many WBPH-resistant varieties and lines had been identified, few have been used in resistance breeding projects, or for detecting resistance genes. This may be because WBPH infestation has not yet paid sufficient attention. Another reason for the poor progress in these areas is due to difficulties in evaluating WBPH resistances using the traditional insect resistance quantification methods. With the increasing risks posed by GSV and RSV infection, it has recently become essential and urgent to identify WBPH resistant germplasm and genes or QTLs to drive pest control research. In this study, we have identified several WBPH-resistant varieties that have been verified with insects collected from Nanning, Guangxi province, and can be used for resistant rice breeding programs.

A total of eight major WBPH resistance genes have been identified from rice varieties or wild rice species to date. Interestingly, $W b p h 7(t)$ and $W b p h 8(t)$ were suggested to be the same loci as Bph14 and Bph15, respectively (Tan et al, 2004), indicating resistance to both WBPH and BPH. Many additional varieties or lines have also been shown to be resistant to both WBPH and BPH (Cao et al, 1993; Huang et al, 2012). Several cloned BPH resistance genes (Bph3, Bph6) have also been proven to confer resistance to both these planthoppers, suggesting there could share mechanisms of resistance in some of these plants. Interestingly, variety N22 was susceptible to $\mathrm{BPH}$, and $\mathrm{OB} 677$ was moderately resistant to $\mathrm{BPH}$, but both were moderately resistant to WBPH (Table 1). These results suggested that insect populations probably 
could change the resistance level conferred by the resistance genes.

In the present study, we mapped two resistance markers, Wbphl and Wbph9. Wbph1 derived from $\mathrm{N} 22$ was mapped to the long arm of chromosome 2 that harbored markers RM13650 and RM13478. A WBPH resistance QTL that was associated with increased seedling survival rate was identified in the Dongxiang wild species, on the short arm of chromosome 2, flanked by markers RM1285 and RM555 derived (Chen et al, 2010). We also showed that Wbph9 was on chromosome 3, flanked by markers RM3513 and RM3525. Wbph7(t) was also mapped to chromosome 3, in a region that harbored markers R1925 and G1318 (Tan et al, 2004). However, these regions were downstream of Wbph9, according to the marker locations. Sogawa et al. (2005) detected several QTLs on chromosomes 2 and 3 that were associated with immigrant density of WBPH or seedling mortality. However, the probable physical positions of these QTLs were different from that of Wbphl and Wbph9 described in our study. Taken together, we believe that both Wbph1 and Wbph9 are novel and unique, and do not correspond to existing genes or QTLs.

Several complementary methods have been used to evaluate WBPH resistance, including the bulk seedling test, honeydew excretion weight, and hatched nymph numbers to identify genes and QTLs associated with resistance. For example, Sogawa et al. (2005) detected five QTLs associated with honeydew excretion weight on chromosomes 2, 3, and 4. Chen et al. (2010) detected three QTLs on chromosomes 2, 5, and 9 from the Dongxiang wild rice species that were associated with seedling survival rate. These discoveries suggest that one gene or QTL can confers multiple traits, or multiple resistant genes or QTLs could regulate a single trait. In the present study, Wbph1 and Wbph9 were respectively identified from N22 and OB677 using the honeydew excretion weight method. It is possible that both genes could also confer resistance when assessed based on other phenotypes, including seedling survival rate or insect development. Indeed, the influence conferred by Wbphl and Wbph9 on seedling survival rates of $\mathrm{N} 22(83.6 \%)$ and OB677 (74.7\%) were partially verified (Fig. 1) and the numbers of hatched insects on N22 (8) and OB677 (11) were also influenced by the presence of these genes (Table 1). 
The scores of LOD and PEV reflect the genetic effects conferred by the genes/QTLs in one mapping population. In the present study, the LOD scores of Wbph1 and Wbph9 were 3.94 and 3.4, respectively, and the respective PEV were $16.6 \%$ and $17.2 \%$, which are generally considered to be low (Table 2). Similar results were reported by Chen et al. (2010), who showed that the PEV of three QTLs ranged from $6.5 \%$ to $8.0 \%$. However, Sogawa et al. (2005) suggested the PEV of three QTLs $q I M G-4, q H N D-4$, and $q E G N-4$ were $78.4 \%, 71.7 \%$, and $58.7 \%$, respectively. To explore the reasons for the diversity of these PEVs, improved evaluation methods are required, to obtain more objective phenotypic scores. Larger mapping populations for gene mapping studies may also improve the PEV. Finally, the detected genes or QTLs Conclusions

The rice varieties PTB33, N22, RBPH327, and OB677 showed resistance to WBPH, of which OB677 was a novel resistance germplasm. Gene mapping indicated that Wbph1 was mapped on chromosome 2 of $\mathrm{N} 22$ in a region harbored with the markers RM3525. chromosome 3 of OB677, where it was flanked by the markers RM3513 and

\section{Abbreviations}

WBPH: whitebacked planthopper; GSV: grassy stunt viruses; RSV: ragged stunt viruses; SRBSDV: Southern rice black-streaked dwarf virus; MAS: marker-assisted selection; LOD: logarithm of odds; SSR: simple sequence repeat; InDel: Insertion/Deletion; BSA: bulked segregant analysis; PCR: polymerase chain reaction; QTL: quantitative trait locus; ANOVA: Analysis of Variance

\section{Ethical Approval and Consent to participate}

Not applicable

\section{Consent for publication}

Not applicable 


\section{Availability of supporting data}

346 Not applicable

\section{Competing interests}

348 The authors declare no competing financial interests.

\section{$349 \quad$ Funding}

350 This research was supported by the National Natural Science Foundation of China 351 (31360325), the Guangxi Natural Science Foundation (2020GXNSFAA259041), the

352 Guangxi Key Laboratory of Biology for Crop Diseases and Insect Pests (2020-KF-02), 353 and the Guangxi Key Laboratory of Rice Genetics and Breeding program 354 (2018-15-Z06-KF11).

\section{Authors' contributions}

356 Conceived and designed the experiments: QY and WB. Performed the experiments:

357 CL, NB, and XA. Analyzed the data: CL, NB, and HW. Wrote, Revised, and approved 358 the final version of the manuscript: QY, WB, CL, and NB. All authors read and 359 approved the final manuscript.

360 Acknowledgements

361 We thank Editage experts for their help with professional English language editing 362 services.

\section{Authors' information}

3641 College of Agriculture, Yangtze University, Jingzhou 434025, China

3652 Plant Protection Institute, Guangxi Key Laboratory of Biology for Crop Diseases 366 and Insect Pests, Guangxi Academy of Agricultural Science, Nanning 530007, China

3673 Rice Research Institute, Guangxi Key Laboratory of Rice Genetics and Breeding, 368 Guangxi Academy of Agricultural Science, Nanning 530007, China

3694 Agricultural College, Guangxi University, Nanning 530004, China 


\section{References}

Angeles ER, Khush GS, Heinrichs EA. New genes for resistance to whitebacked planthopper in rice. Crop Sci. 1981;21: 47-50.

Athwal DS, Pathak MD, Bacalangco EH, Pura CD. Genetics of resistance to brown planthoppers and green leafhoppers in Oryza sativa L. Crop Sci. 1971;11: 747-750.

Cao YS, Zhang XZ, Huang QG. Merit rice germplasm information in national germplasm resource data library. Crop Var Resour. 1993;3: 19-21 (In Chinese with English abstract).

Chen J, Huang DR, Wang L, Liu GJ, Zhuang JY. Identification of quantitative trait loci for resistance to whitebacked planthopper, Sogatella furcifera, from an interspecific cross Oryza sativa $\times$ O.rufipogon. Breed Sci. 2010;60(2): 153-159.

Du B, Zhang WL, Liu BF, Hu J, Wei Z, Shi ZY, He RF, Zhu LL, Chen RZ, Han B, He GC. Identification and characterization of $B p h 14$, a gene conferring resistance to brown planthopper in rice. Proc Natl Acad Sci USA. 2009;106(52): 2163-22168.

Du B, Chen RZ, Guo JP, He GC. Current understanding of the genomic, genetic, and molecular control of insect resistance in rice. Mol Breed. 2020;40: 24.

Hernandez JE, Khush GS. Genetics of resistance to whitebacked planthopper in some rice (Oryza sativa L.) varieties. Oryza. 1981;18: 44-50.

Huang FK, Huang SS, Wu BQ, Wei S M. Screening of rice germplasms resistant to brown planthopper (BPH) and white-backed planthopper (WBPH). Plant Prot 2012;38(4): 152-155 (In Chinese with English abstract).

Hu SJ, Liu XF, Fu DY, Huang W, Wang XY, Liu XJ, Lv JP, Ye H. Projecting distribution of the overwintering population of Sogatella furcifera (Hemiptera: Delphacidae), in Yunnan, China with analysis on key influencing climatic factors. J Insect Sci. 2015;15: 148.

Khush GS, Rezaul Karim ANM, Angeles ER. Genetic analysis of rice cultivar ARC 10550 to Bangladesh brown planthopper biotype. J Genet. 1985:64: 121-125.

Lakshminarayana A, Khush GS. New genes for resistance to the brown planthopper in rice. Crop Sci. 1977; 17: 96-100.

Zhou GH, Xu GL, Xu DG, Zhang MX. Southern rice black-streaked dwarf virus: a 
white-backed planthopper-transmitted fijivirus threatening rice production in Asia. Front Microbiol. 2013;4: 270.

Li XM, Xiong ZM, Min SK, Hu GW. Genetical analysis of resistance to whitebacked planthopper Sogatella furcifera (Horvath) in four rice varieties (Oryza sativa L.) of Yunan province. Chin J Rice Sci. 1990;4: 113-116.

Li XM, Zhai HQ, Wan JM. Preliminary mapping of $\operatorname{Wbph} 6(t)$ a new gene resistant to whitebacked planthopper in rice. Rice Sci. 2004;11(3): 86-90.

Li ZH, Xue YX, Zhou HL, Li Y, Usman B, Jiao XZ, Wang XY, Liu F, Qin BX, Li RB, Qiu YF. High-resolution mapping and breeding application of a novel brown planthopper resistance gene derived from wild rice (Oryza. rufipogon Griff). Rice. 2019;12: 41.

Liu ZY, Liu GJ, Kazushige S, Zhuang JY, Chen SG, Shen JH, Zheng KL. Mapping the gene Wbph2 in ARC10239 resistant to the whitebacked planthopper, Sogatella furcifera in rice. Chin J Rice Sci. 2002:16(4): 311-314 (Chinese with English abstract).

McCouch SR, Khush GS, Tanksley SD. Tagging genes for disease and insect resistance via linkage to RFLP markers. Rice Genetics II. Manila, Philippines, 1991; 1991: 443-449.

Nemoto H, Ikeda R, Kaneda C. New genes for resistance to brown planthopper, Nilaparvata lugens Stål, in rice. Jpn J Breed. 1989;39: 23-28.

Qiu YF, Cheng L, Zhou P, Liu F, Li RB. Identification of antixenosis and antibiosis in two newly explored brown planthopper-resistance rice lines. Adv J Food Sci and Technol. 2012;4(5): 299-303.

Qiu Y, Guo J, Jing S, Zhu L, He G. High-resolution mapping of the brown planthopper resistance gene $B p h 6$ in rice and characterizing its resistance in the 9311 and Nipponbare near isogenic backgrounds. Theor Appl Genet. 2010;121(8): $1601-1611$.

Qiu Y, Guo J, Jing S, Tang M, Zhu L, He G. Identification of antibiosis and tolerance in rice varieties carrying brown planthopper resistance genes. Entomol Exp Appl. $2011 ; 141: 224-231$. 
431 Sidhu GS, Khush GS, Medrano FG. A dominant gene in rice for resistance to

432 whitebacked planthopper and its relationship to other plant characteristics.

$433 \quad$ Euphytica. 1979;28: 227-232.

434 Sogawa K, Qian Q, Zeng D, Hu J, Zeng LJ. Differential expression of whitebacked

435 planthopper resistance in the Japonica /Indica doubled haploid rice population

436 under field evaluation and seedbox screening test. Rice Sci. 2005;12(1): 63-67.

437 Tan GX, Weng QM, Ren X, Huang Z, Zhu LL, He GC. Two whitebacked planthopper

438 resistance genes in rice share the same loci with those for brown planthopper

439 resistance. Heredity. 2004;92: 212-217.

$440 \mathrm{Wu}$ CF, Khush GS. A new dominant gene for resistance to whitebacked planthopper

441 in rice. Crop Sci. 1985;25: 505-509.

442 Yamazaki Y, Tsuchiya R, Takahashi Y, Asanuma T, Shidahara Y, Sakaniwa S. Rice

443 genes in Oryza base. Nat Preced. 2010;doi:10.1038/npre.2010.5069.1. 


\section{Figure 1:}
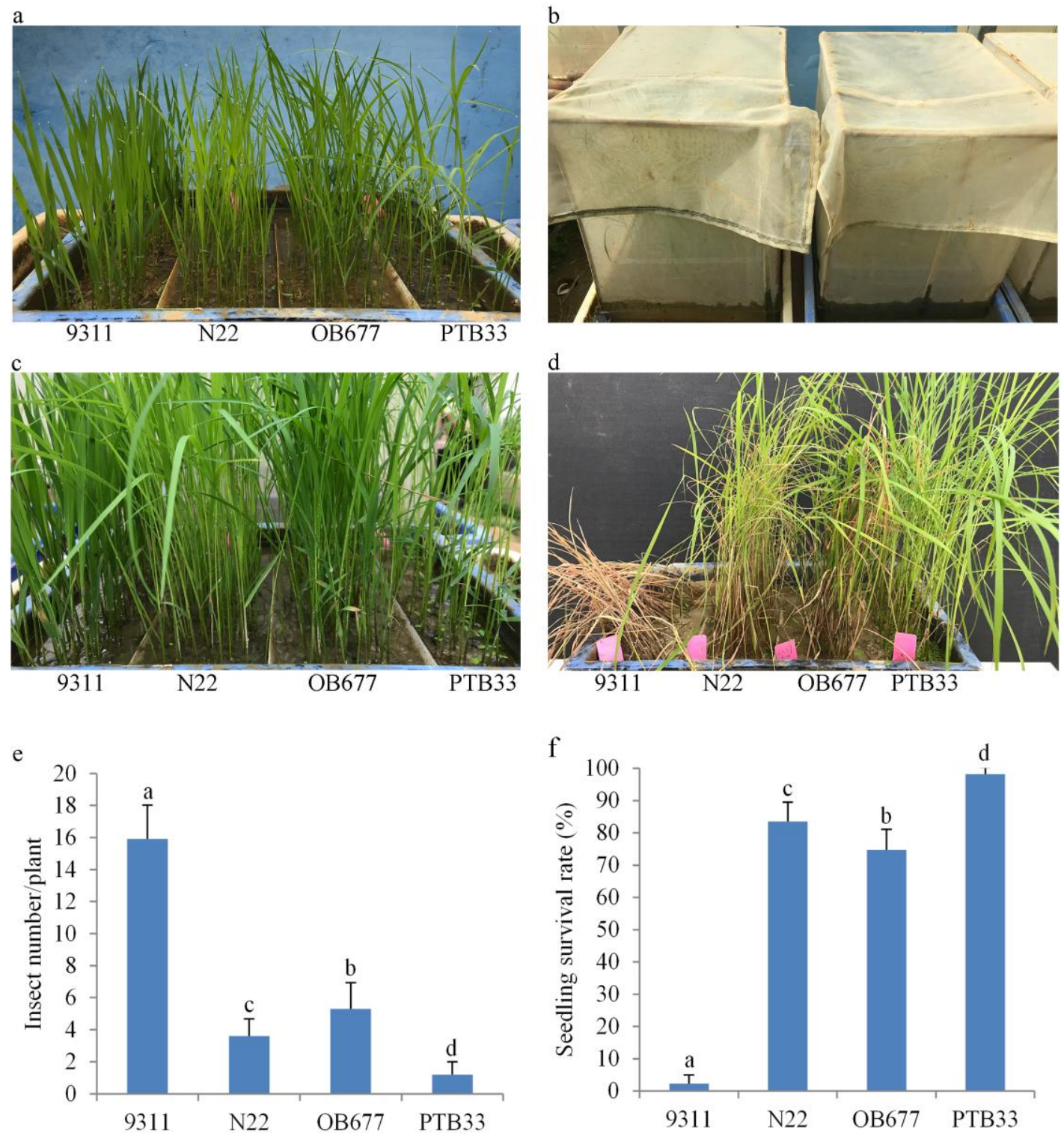

Fig. 1 Detection of WBPH host choice and seedling survival rate. (a, b) Photographs depicting general infestation with WBPH and at (c) 10 days and (d) 30 days after infestation. (e) Numbers of insects per plant and (f) seedling survival rate across the four indicated plant varieties.

Fig. 2 Distribution of honeydew excretion weight per WBPH per $48 \mathrm{~h}$ after feeding on the $\mathrm{F}_{2}$ mapping populations (a) 9311/N22 and (b) 9311/OB677. 
455 Table 1 Evaluation of insect resistance of selected varieties of rice plants.

\begin{tabular}{|c|c|c|c|c|c|}
\hline Variety & $\begin{array}{l}\text { Resistance } \\
\text { gene }\end{array}$ & $\begin{array}{l}\text { WBPH } \\
\text { resistance } \\
\text { score }\end{array}$ & $\begin{array}{l}\text { WBPH } \\
\text { Honeydew } \\
\text { excretion }(\mathrm{mg} / 2 \\
\text { days })\end{array}$ & $\begin{array}{l}\text { WBPH } \\
\text { No. of nymphs }\end{array}$ & $\begin{array}{l}\mathrm{BPH} \\
\text { resistance } \\
\text { score }\end{array}$ \\
\hline $\mathrm{N} 22$ & Wbphl & $4.2 \pm 0.6^{\mathrm{a}}$ & $1.4 \pm 0.7^{b}$ & $8 \pm 2^{\mathrm{a}}$ & $7.2 \pm 0.5^{\mathrm{d}}$ \\
\hline $\begin{array}{l}\text { Baiganru } \\
\text { o }\end{array}$ & Wbph2 & $6.2 \pm 0.7^{b}$ & $6.0 \pm 2.2^{\mathrm{cd}}$ & $14 \pm 3^{\mathrm{a}, \mathrm{b}}$ & $8.2 \pm 0.6^{\mathrm{e}}$ \\
\hline Nebeshi & Wbph3 & $7.1 \pm 1.1^{\mathrm{c}}$ & $6.2 \pm 1.6^{\mathrm{cd}}$ & $32 \pm 10^{\mathrm{c}}$ & $7.3 \pm 1.3^{\mathrm{d}}$ \\
\hline Cdombo & wbph4 & $6.6 \pm 0.9 b^{c}$ & $4.6 \pm 1.8^{c}$ & $12 \pm 5^{\mathrm{ab}}$ & $7.6 \pm 1.2^{\mathrm{d}}$ \\
\hline Mudgo & Bphl & $7.9 \pm 1.2 c^{d}$ & $4.8 \pm 2.3^{c}$ & $28 \pm 9^{c}$ & $7.9 \pm 0.9^{\mathrm{d}, \mathrm{e}}$ \\
\hline ASD7 & $b p h 2$ & $8.1 \pm 0.5^{\mathrm{c}, \mathrm{d}}$ & $8.4 \pm 2.2^{\mathrm{d}}$ & $36 \pm 8^{\mathrm{c}}$ & $8.2 \pm 0.7^{\mathrm{e}}$ \\
\hline PTB33 & Bph3 & $3.2 \pm 0.6^{\mathrm{a}}$ & $0.2 \pm 0.03^{\mathrm{a}}$ & $5 \pm 2^{\mathrm{a}}$ & $2.7 \pm 0.4^{\mathrm{a}}$ \\
\hline $\begin{array}{l}\text { Babawee } \\
\text { ARC105 }\end{array}$ & bph4 & $4.6 \pm 0.7^{\mathrm{a}, \mathrm{b}}$ & $1.2 \pm 0.7^{\mathrm{b}}$ & $6 \pm 2^{\mathrm{a}}$ & $3.6 \pm 0.7^{\mathrm{a}, \mathrm{b}}$ \\
\hline 50 & bph5 & $8.1 \pm 0.8^{\mathrm{c}, \mathrm{d}}$ & $6.8 \pm 2.1^{\mathrm{c}, \mathrm{d}}$ & $32 \pm 9^{c}$ & $8.3 \pm 0.4^{\mathrm{e}}$ \\
\hline RBPH16 & $\begin{array}{l}\text { Bph27+ } \\
\text { Bph36 }\end{array}$ & $5.2 \pm 0.9^{\mathrm{a}, \mathrm{b}}$ & $2.2 \pm 0.8^{b}$ & $9 \pm 2^{a}$ & $3.4 \pm 0.6^{\mathrm{a}, \mathrm{b}}$ \\
\hline $\begin{array}{l}\text { RBPH32 } \\
7\end{array}$ & Unknown & $4.0 \pm 0.6^{\mathrm{a}}$ & $2.1 \pm 0.6^{\mathrm{b}}$ & $16 \pm 6^{\mathrm{a}, \mathrm{b}}$ & $3.5 \pm 0.8^{\mathrm{a}, \mathrm{b}}$ \\
\hline OB677 & Unknown & $3.8 \pm 0.8^{\mathrm{a}}$ & $1.2 \pm 0.4^{\mathrm{b}}$ & $11 \pm 4^{\mathrm{a}, \mathrm{b}}$ & $5.2 \pm 0.7^{\mathrm{c}}$ \\
\hline 9311 & None & $8.5 \pm 0.7^{\mathrm{d}}$ & $12.6 \pm 3.2^{\mathrm{e}}$ & $52 \pm 11^{\mathrm{d}}$ & $8.7 \pm 0.3^{f}$ \\
\hline TN1 & None & $8.7 \pm 0.3^{\mathrm{d}}$ & $9.2 \pm 2.8 \mathrm{~d}$ & $50 \pm 7^{\mathrm{d}}$ & $8.9 \pm 0.4^{\mathrm{f}}$ \\
\hline
\end{tabular}

456 The same letter within a column are not significantly different (least significant

457 difference test: $\mathrm{P}>0.05)$. 
Table 2 Mapping of WBPH resistance genes

\begin{tabular}{llllll}
\hline Chr.2 & Position (cM) & LOD Score & PEV $(\%)^{1}$ & Additive $^{2}$ & Dominant \\
\hline 2M021 & 0 & 1.53 & 6.7 & -1.7 & -2.0 \\
RM530 & 7.4 & 2.71 & 11.6 & -2.7 & -1.9 \\
RM13650 & 13.5 & 3.84 & 16.2 & -3.2 & -2.4 \\
Wbph1 & 14.5 & 3.94 & 16.6 & -3.3 & -2.4 \\
RM13478 & 14.6 & 3.92 & 16.5 & -3.3 & -2.4 \\
2M15.039 & 21.1 & 2.33 & 10.2 & -2.3 & -2.7 \\
\hline
\end{tabular}

Chr.3

\begin{tabular}{llllll} 
RM231 & 0 & 1.7 & 10.1 & -0.4 & -0.4 \\
RM7395 & 4.8 & 2.4 & 12.8 & -0.5 & -0.2 \\
RM3513 & 6.2 & 3.0 & 15.2 & -0.5 & -0.3 \\
Wbph9 & 6.4 & 3.4 & 17.2 & -0.5 & -0.3 \\
RM3525 & 6.6 & 3.3 & 16.4 & -0.5 & -0.3 \\
3E51292 & 11.6 & 1.2 & 7.2 & -0.3 & -0.3 \\
\hline
\end{tabular}

$461{ }^{1}$ The percentage of total phenotypic variance (PEV \%) was contributed by the locus.

$462{ }^{2}$ Additive means additive effect of the associated marker indicated from 9311;

463 dominant means dominant effect of the associated marker indicated from 9311. 


\section{Figures}
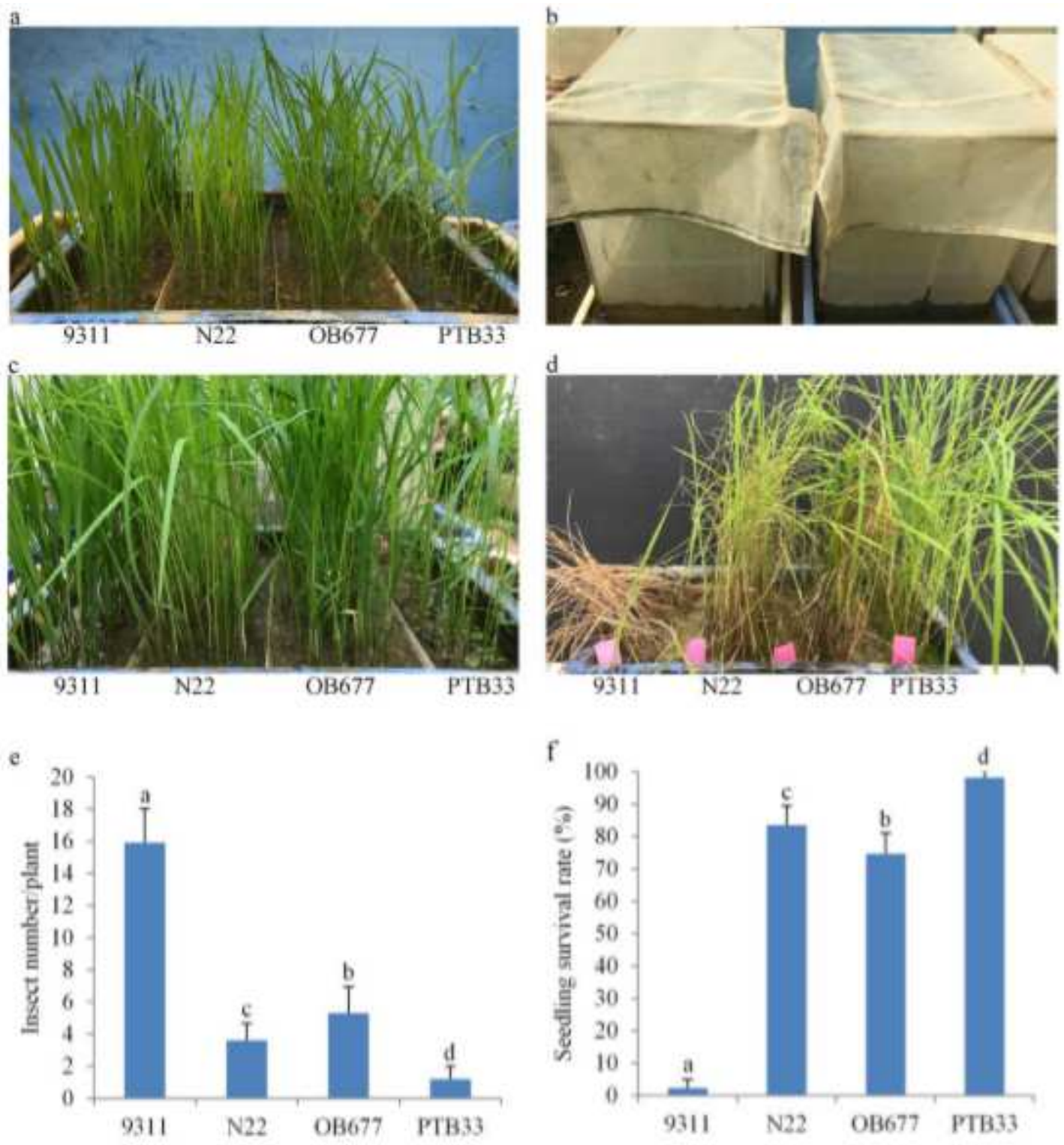

\section{Figure 1}

Detection of WBPH host choice and seedling survival rate. $(a, b)$ Photographs depicting general infestation with WBPH and at (c) 10 days and (d) 30 days after infestation. (e) Numbers of insects per plant and (f) seedling survival rate across the four indicated plant varieties. 

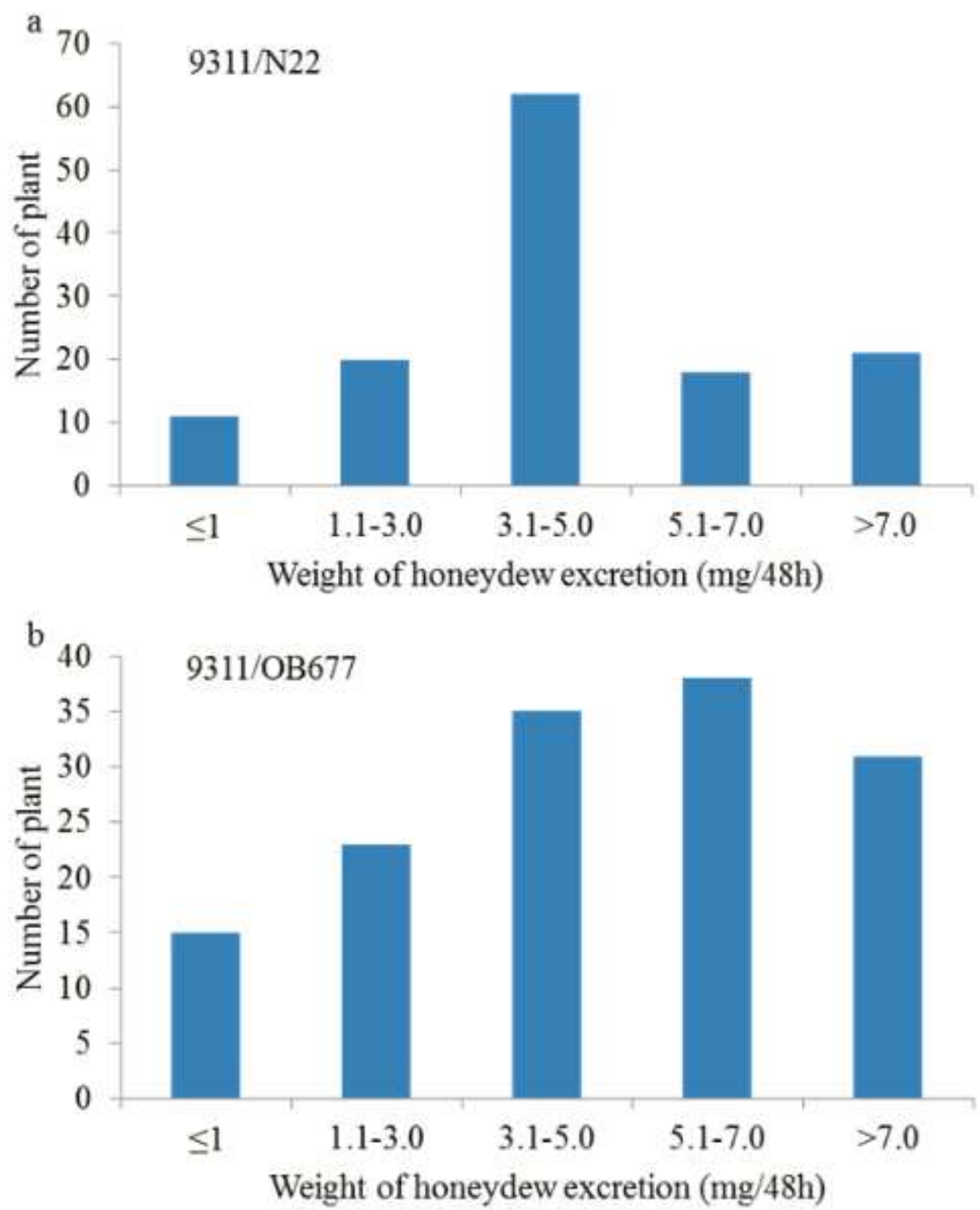

Figure 2

Distribution of honeydew excretion weight per WBPH per $48 \mathrm{~h}$ after feeding on the F2 mapping populations (a) 9311/N22 and (b) 9311/OB677. 